\title{
SOME REMARKS ON UNIQUENESS FOR A CLASS OF SINGULAR ABSTRACT CAUCHY PROBLEMS
}

\author{
JAMES A. DONALDSON ${ }^{1}$ AND JEROME A. GOLDSTEIN
}

\begin{abstract}
Of concern is the Cauchy problem for equations of the form $u^{\prime \prime}(t)+\alpha(t) u^{\prime}(t)+S^{2} u(t)=0\left(^{\prime}=d / d t\right)$ on a complex Hilbert space $X . S$ is a selfadjoint operator on $X$ while $\alpha$ is a continuous function on $(0, \infty)$ which can be unbounded at $t=0$. Uniqueness results are obtained for these equations by applying a uniqueness theorem for nonlinear equations. Furthermore, nonuniqueness examples for the linear abstract Euler-PoissonDarboux equation, which is contained in this class, show that the uniqueness theorem is best possible.
\end{abstract}

Introduction. We shall consider the linear evolution equation

$$
u^{\prime}(t)=(A+P(t)) u(t) \quad(t>0)
$$

in a Banach space $X$. Here prime denotes differentiation with respect to $t$. Let $A$ be the infinitesimal generator of a $\left(C_{0}\right)$ semigroup of linear operators on a Banach space $X$. (We use the standard notation and terminology of operator semigroup theory; cf. Hille and Phillips [13], Goldstein [11].) Let $P$ be a strongly continuously differentiable function from $(0, \infty)$ to the bounded linear operators on $X$.

Definition 1. A type (I) solution of (1) is a function $u \in C^{1}([0, \infty) ; X)$ satisfying (1) for $t \in(0, \infty)$.

Definition 2. A type (II) solution of (1) is a function $u \in C^{1}((0, \infty) ; X)$ $\cap C([0, \infty) ; X)$ satisfying $(1)$ for $t \in(0, \infty)$.

Note that a type (I) solution is clearly also a type (II) solution. The converse, however, is false, as will be seen below.

Together with equation (1) we shall consider the initial condition

$$
u(0)=f \in \mathscr{D}(A) ;
$$

$\mathcal{D}(A)$ denotes the domain of $A$. A uniqueness theorem for type (I) solutions of (1), (2) is given by the following consequence of the main result of Goldstein's paper [10].

Received by the editors December 3, 1974.

AMS (MOS) subject classifications (1970). Primary 35Q05, 34G05, 35G10; Secondary 47A50, 47B44, 47F05.

Key words and phrases. Singular Cauchy problem, uniqueness, dissipative operators, EPD equation.

${ }^{1}$ Supported in part by NSF Grant No. GY-11066. 
THEOREM 1. Suppose $P(t)-K(t) I$ is dissipative for each $t>0$ where $K(t)=t^{-1}+b$ for some real number $b$. Then the initial value problem (1), (2) has at most one type I solution.

Proof. By replacing the norm on $X$ by an equivalent norm we may assume that $A-a I$ is dissipative for some real number $a$ (cf. [13, p. 364], [11, p. 11]). Let $u_{1}, u_{2}$ be two type (I) solutions of (1), (2). Then for $j=1,2, v_{j}(t)$ $=e^{-(a+b) t} u_{j}(t)$ is a type (I) solution of

$$
\begin{aligned}
& v^{\prime}(t)=[A+P(t)-(a+b) I] v(t) \quad(t>0), \\
& v(0)=f .
\end{aligned}
$$

Since $[A+P(t)-(a+b) I]-t^{-1} I$ is dissipative for each $t>0, v_{1} \equiv v_{2}$ follows from Theorem 1 of [10]. Hence $u_{1} \equiv u_{2}$.

A uniqueness result for second order Cauchy problems. Now let $S$ be a selfadjoint operator on a complex Hilbert space $Y$. (More generally, we could replace $i S$ by $B$, the infinitesimal generator of a $\left(C_{0}\right)$ group acting on a real or complex Banach space $Y$.) Consider now the abstract differential equation

$$
v^{\prime \prime}(t)+\alpha(t) v^{\prime}(t)+S^{2} v(t)=0 \quad(t>0)
$$

with initial conditions

$$
v(0)=\phi \in \mathscr{Q}\left(S^{2}\right), \quad v^{\prime}(0)=0 .
$$

Since $\alpha(t)$ is allowed to be unbounded at $t=0$, the class of Cauchy problems under investigation here may be singular. Now let

$$
\begin{aligned}
u(t) & =\left(\begin{array}{c}
v(t) \\
v^{\prime}(t)
\end{array}\right), & A & =\left(\begin{array}{cc}
0 & I \\
-S^{2} & 0
\end{array}\right), \\
P(t) & =\left(\begin{array}{cc}
0 & 0 \\
0 & -\alpha(t) I
\end{array}\right), & f & =\left(\begin{array}{l}
\phi \\
0
\end{array}\right) ;
\end{aligned}
$$

and let $X$ be the completion of $\mathscr{D}(S) \times Y$ under the norm $\left\|\left(\begin{array}{l}x \\ y\end{array}\right)\right\|$ $=\left\{|S x|^{2}+|y|^{2}\right\}^{1 / 2}$, where $|\cdot|$ denotes the norm in $Y$. Then $i A$ (with domain $\left.\mathscr{D}\left(S^{2}\right) \times \mathscr{D}(S)\right)$ is a selfadjoint operator on the Hilbert space $X$. (1) [resp. (2)] is equivalent to (3) [resp. (4)] under the identification (5). In particular, $u$ is a type (I) [resp. type (II)] solution of (1) if and only if $v$ satisfies (3) for $t \in(0, \infty)$ and $v \in C^{2}([0, \infty) ; Y) \quad\left[v \in C^{1}([0, \infty) ; Y) \cap C^{2}((0, \infty) ; Y)\right]$. In this case we shall call $v$ a type (I) [resp. type (II)] solution of (3).

THEOREM 2. The initial value problem (3), (4) has at most one type (I) solution if $\alpha(t) \geqq-1 / t$ for all $t>0$.

Proof. $P(t)-K_{1}(t) I$ is dissipative for each $t>0$, where $P(t)$ is given by (5) and $K_{1}(t)=-1 / t$. This can be readily seen by computing

$$
\operatorname{Re}\left\langle\left[P(t)-K_{1}(t) I\right]\left(\begin{array}{l}
x \\
y
\end{array}\right),\left(\begin{array}{l}
x \\
y
\end{array}\right)\right\rangle=-t^{-1}\langle S x, S x\rangle-\left(\alpha(t)+t^{-1}\right)\langle y, y\rangle ;
$$


this is clearly nonpositive whenever $\alpha(t) \geqq-t^{-1}$. Consequently, for $\alpha(t)$ $\geqq-t^{-1}$, the conclusion of Theorem 2 is an immediate consequence of Theorem 1.

REMARK. For $\alpha(t) \equiv 0$ we obtain the well-known result of Hille [12] that the Cauchy problem for the abstract wave equation can have at most one type (I) solution.

The abstract Euler-Poisson-Darboux equation. We apply the theory developed in the preceding section to the Cauchy problem for the abstract EulerPoisson-Darboux (EPD) equation

$$
\begin{gathered}
u^{\prime \prime}(t)+(\rho / t) u^{\prime}(t)+S^{2} u(t)=0, \\
u(0)=\phi, \quad \phi \in \mathscr{D}\left(S^{2}\right), \quad u^{\prime}(0)=0,
\end{gathered}
$$

and obtain uniqueness results for this problem. This problem is singular for $\rho \neq 0$ since the initial data is defined at $t=0$ where a coefficient of the equation becomes unbounded. Recently the EPD equation has received much attention in the literature; see Carroll [2]-[5], Donaldson [8], [9] and Lions [14]. Its concrete analogue

$$
\begin{gathered}
u_{t t}+(\rho / t) u_{t}-u_{x x}=0, \\
u(x, 0)=\phi(x), \quad u_{t}(x, 0)=0
\end{gathered}
$$

is classical, and the fundamental work of Weinstein was a catalyst for recent investigations of this equation. In this regard we mention the work of Blum [1], Diaz and Weinberger [7], Delache and Leray [6], Tersenov [15], and Weinstein [16]-[19]. Also, see the references in [19].

We now state the following uniqueness result for the Cauchy problem for the abstract EPD equation.

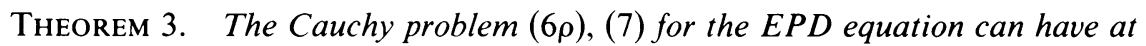
most one type (I) solution for $\rho \geqq-1$.

Proof. This is an immediate consequence of Theorem 2 since $\rho t^{-1}$ $\geqq-t^{-1}$ for $\rho \geqq-1$.

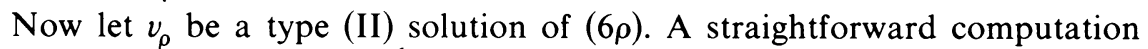
shows that $w_{\rho}(t)=v_{\rho}(t)+t^{1-\rho} v_{2-\rho}(t)$ is a type (II) solution of $(6 \rho)$ for $\rho<0$ and $\rho$ not a negative odd integer. In particular, $u_{\rho}(t)=t^{1-\rho} v_{2-\rho}(t)$ satisfies $(6 \rho)$ for $\rho<0$ with $\phi=0$ in (7). For $q>0$, a type (I) solution to (6q) and (7) (with $\phi=\psi \in \mathcal{D}\left(S^{2}\right)$ ) is given by

$$
v_{q}(t)=c_{q} \int_{-1}^{1}\left(1-\sigma^{2}\right)^{q / 2-1} \exp \{i \sigma t S\} \psi d \sigma
$$

where $c_{q}=\Gamma((q+1) / 2) /\{\Gamma(q / 2) \Gamma(1 / 2)\}$. (See Donaldson [8], [9].)

THEOREM 4. Nonuniqueness of type (II) solutions of (6o), (7) holds when $\rho<0$ and $\rho$ is not a negative odd integer.

Proof. Let $\phi=0$ in (7). Let $0 \neq \psi \in \mathcal{D}\left(S^{m+2}\right)$ where $m$ is the smallest 
positive integer such that $2 m+\rho>0$. Let $q=2-\rho>0$ and let $v_{q}$ be defined by $(10 q)$. Then $v_{\rho} \equiv 0$ and $u_{\rho}(t) \equiv t^{1-\rho} v_{q}(t)$ are distinct type (II) solutions of $(6 \rho)$ satisfying zero initial conditions.

In the above proof $v_{\rho}$ is clearly a type (I) solution. Also, $u_{\rho}$ is a type (I) solution if $\rho<-1$ since a straightforward computation shows that $v_{\rho}^{\prime \prime}(t)$ exists at $t=0$. This shows that Theorem 1 is a sharp result in the following sense.

THEOREM 5. The conclusion in Theorem 1 becomes false if the hypothesis on $K(t)$ is changed to $K(t)=(1+\epsilon) t^{-1}+b$ for some real $b$ and some $\epsilon>0$.

Of course, if $K(t)$ is replaced by $(1-\epsilon) t^{-1}+b$ for some real $b$ and some $\epsilon>0$, then the hypothesis of Theorem 1 is satisfied, so we have uniqueness of type (I) solutions of the Cauchy problem (1), (2).

REMARKS. 1. In [10] it was pointed out that a nonlinear example of Perron showed that the largest permissible constant $c$ in $K(t)=c t^{-1}$ was $c=1$. The above results for the abstract EPD equation show that the same is true in the context of the linear theory.

2. Uniqueness of the solution of the initial value problem $(8 \rho),(9)$ for $\rho>0$ has been established by Asgeirsson. However, for $\rho<0$ there can be no uniqueness, since any function of the type $t^{1-\rho} u_{2-\rho}(x, t)$, which vanishes together with its derivative with respect to $t$ at $t=0$, may be added to a solution $u_{\rho}(x, t)$ of the Cauchy problem. The sum is also a solution.

3. For the concrete problem $(8 \rho),(9)$ it is true that the EPD equation is singular for all values of $\rho \neq 0$. However, it is only when $\rho$ is a negative odd integer that one obtains singular behavior at $t=0$. Unless the initial values are sufficiently smooth, the derivatives of $u_{\rho}(x, t)$ of a certain order with respect to $t$ will become infinite for $t=0$ (cf. [18], [6], [1]).

4. One can obtain nonuniqueness of type (II) solutions of $(6 \rho)$, (7) when $\rho$ is a negative odd integer by employing a modification of the work of Blum [1].

\section{REFERENCES}

1. E. K. Blum, The Euler-Poisson-Darboux equation in the exceptional cases, Proc. Amer. Math. Soc. 5 (1954), 511-520. MR 16, 137.

2. R. W. Carroll, On the singular Cauchy problem, J. Math. Mech. 12 (1963), 69-102. MR 26 \# 5284.

3. _- Some singular Cauchy problems, Ann. Mat. Pura Appl. (4) 56 (1961), 1-31. MR 27 \#1706.

4. —- Quelques problèmes de Cauchy singuliers, C. R. Acad. Sci. Paris 251 (1960), 498-500. MR 22 \#3877.

5. —- Some singular mixed problems, Proc. Nat. Acad. Sci. U. S. A. 46 (1960), 1594-1596. MR 24 \# A 1522.

6. S. Delache and J. Leray, Calcul de la solution élémentaire de l'opérateur d'Euler-PoissonDarboux et de l'opérateur de Tricomi-Clairaut, hyperbolique, d'ordre 2, Bull. Soc. Math. France 99 (1971), 313-336. MR 46 \#2261.

7. J. B. Diaz and H. F. Weinberger, A solution of the singular initial value problem for the EulerPoisson-Darboux equation, Proc. Amer. Math. Soc. 4 (1953), 703-715. MR 15, 321.

8. J. A. Donaldson, A singular abstract Cauchy problem, Proc. Nat. Acad. Sci. U. S. A. 66 (1970), 269-274. MR 42 \# 706.

9. _- An operational calculus for a class of abstract operator equations, J. Math. Anal. Appl. 37 (1972), 167-184. MR 45 \#753.

10. J. A. Goldstein, Uniqueness for nonlinear Cauchy problems in Banach spaces, Proc. Amer. Math. Soc. 53 (1975), 91-95. 
11. J. A. Goldstein, Semigroups of operators and abstract Cauchy problems, Lecture Notes, Department of Mathematics, Tulane University, New Orleans, La., 1970.

12. E. Hille, Une généralisation du problème de Cauchy, Ann. Inst. Fourier (Grenoble) 4 (1952), 31-48 (1954). MR 15, 718.

13. E. Hille and R. S. Phillips, Functional analysis and semigroups, rev. ed., Amer. Math. Soc. Colloq. Publ., vol. 31, Amer. Math. Soc., Providence, R. I., 1957. MR 19, 664.

14. J.-L. Lions, Opérateurs de transmutation singuliers et équations d'Euler-Poisson-Darboux généralisées, Rend. Sem. Mat. Fis. Milano 28 (1959), 124-137. MR 22 \# 4882.

15. S. A. Tersenov, On a singular Cauchy problem, Dokl. Akad. Nauk SSSR 196 (1971), 1032-1035 = Soviet Math. Dokl. 12 (1971), 326-330. MR 43 \#2350.

16. A. Weinstein, On the wave equation and the equation of Euler-Poisson, Proc. Sympos. Appl. Math., vol. 5, Amer. Math. Soc., Providence, R.I., 1954, pp. 137-147. MR 16, 137.

17. - The generalized radiation problem and the Euler-Poisson-Darboux equation, Summa Brasil. Math. 3 (1955), 125-147. MR 17, 1091.

18. _- Sur le problème de Cauchy pour l'équation de Poisson et l'équation des ondes, C. R. Acad. Sci. Paris 234 (1952), 2584-2585. MR 14, 176.

19. - - Singular partial differential equations and their applications, Fluid Dynamics and Applied Mathematics (Proc. Sympos., Univ. of Maryland, 1961), Gordon and Breach, New York, 1962, pp. 29-49. MR 27 \#3926.

Department of Mathematics, Howard University, Washington, D. C. 20059

Department of Mathematics, Tulane University, New Orleans, Louisiana 70118 